\title{
Editorial
}

\section{Medical Application of 3D Printing: A Powerful Tool for Personalised Treatment}

\author{
$D A I$ Kerong $^{\mathrm{a} *}$ (戴攰戎), $X U \mathrm{Feng}^{\mathrm{b}}$ (许 锋) \\ (a. Shanghai Key Laboratory of Orthopaedic Implants, Department of Orthopaedic Surgery; \\ b. Department of Discipline Planning, Technology Transfer Office, Shanghai Ninth People's Hospital, \\ Shanghai Jiao Tong University School of Medicine, Shanghai 200011, China)
}

(C) Shanghai Jiao Tong University and Springer-Verlag GmbH Germany, part of Springer Nature 2021

We are in an era of technological revolutions promoting personalised healthcare. Advances in medical imaging techniques with 3D imaging software and $3 \mathrm{D}$ printing have allowed healthcare professionals to view and document various geometrical structures in a brand-new way, enabling them to make meaningful 3D measurements more accurately by generating both virtual and physical models used for preoperative planning, physician-patient communication, and fabrication of surgical guides, instruments, and implants ${ }^{[1-5]}$. With improvements in cost-effectiveness, efficiency, and mechanical properties, 3D printing technologies have become a powerful tool for physicians to meet clinical requirements. Furthermore, biological tissues made from $3 \mathrm{D}$ printing may eventually provide patients with required human organs in the future ${ }^{[6-7]}$.

Although both medical communities and social media spare no effort to highlight the prospects of $3 \mathrm{D}$ printing technologies in healthcare and popularise this innovative new method through Web-based approaches to promote its application in personalised treatment, to date, not a single healthcare organisation has yet released new technologies, disseminated findings in peerreviewed literature, or clarified the role and aims of $3 \mathrm{D}$ printing in healthcare. This, in turn, has left much of the current research and development to medical device companies, expecting them to meet individual clinical requests. Therefore, at present and in the future, it is critical to have a clear understanding of the clinical implementation of 3D printing for both traditional and personalised healthcare.

The first step in the entire 3D printing process starts with medical imaging, in which professionals with expertise in radiology and imaging processing have con-

\footnotetext{
Received: 2021-03-28 Accepted: 2021-03-28

*E-mail: krdai@163.com
}

ducted many investigations to identify and quantify patient-specific anatomical areas and geometrical structures before designing and manufacturing personalised medical models, surgical guides, medical instruments, and devices using $3 \mathrm{D}$ printing ${ }^{[8-11]}$. It must be noted that $3 \mathrm{D}$ printing usually starts with conventional clinical images, and errors cannot be avoided with several more complex steps involved in image processing. Traditionally, medical images have been acquired in radiology departments by trained radiologists using special software packages. With the help of commercial medical imaging processing software, such as MIMICS, Analyze, and MeDraw, many physicians from different specialties, with little engineering background, are able to perform these analyses by themselves and develop their preoperative planning software tailored to special medical treatments. Understandably, the development of software tailored to 3D printing needed in medicine could accelerate and promote its popularity in clinical practice.

Organised by Journal of Shanghai Jiao Tong University (Science), this special issue, authored by a group of physicians and engineers with diverse and interdisciplinary backgrounds and insights, is intended to introduce their research topics in the most hotly debated areas where medical 3D printing is used in patient care, especially focusing on medical and dental applications. The issue also presents some related topics about imaging generation and processing, material properties, and biomechanics, among others. However, it is difficult to cover all the $3 \mathrm{D}$ printing fundamentals.

In the current special issue, some interesting studies provide details regarding how to apply $3 \mathrm{D}$ printing to medical or dental personalised healthcare, which could be invaluable for physicians who would like to find their own methods of developing personalised routines by applying $3 \mathrm{D}$ printing in daily practice. The 
issue also includes some dedicated studies that focus on imaging and software applications, which are indispensable for those who are eager to enter the field of pre-processing in 3D printing. Last but not least, some studies discussing material properties and biomechanics with in-depth insights regarding the safety and reliability of 3D printing technology in the manufacturing of medical devices can be found in this issue. As authors and advocates of personalised treatment, we are interested in promoting $3 \mathrm{D}$ printing from its current niche applications to more widespread use in the medical community. Thus, this special issue also includes studies on some of these niche applications. Since 3D printing technology is now growing at an exponential rate, it is definitely a very challenging task to organise issues on $3 \mathrm{D}$ printing in personalised patient care. In this issue, we attempt to inspire our readers by choosing some clinical examples in several representative areas to show how 3D printing positively influences personalised healthcare.

There is no doubt that 3D printing is truly one of the leading technologies of the 21st century and praised as a key feature of the fourth industrial revolution. We hope this special issue could provide essential information to help you understand the role that $3 \mathrm{D}$ printing plays in personalised patient care with the purpose of improving clinical outcomes and quality of life for patients in China and around the world. Finally, we genuinely hope that people with lofty ideals from both medical and engineering fields who are interested in 3D printing technologies notice this special issue and join us to enter the field with your meaningful contributions.

\section{References}

[1] DAI K R, YAN M N, ZHU Z A, et al. Computer-aided custom-made hemipelvic prosthesis used in extensive pelvic lesions [J]. The Journal of Arthroplasty, 2007, 22(7): 981-986.
[2] MCGURK M, AMIS A A, POTAMiAnOS P, et al. Rapid prototyping techniques for anatomical modelling in medicine [J]. Annals of the Royal College of Surgeons of England, 1997, 79(3): 169-174.

[3] LI H W, WANG L, MAO Y Q, et al. Revision of complex acetabular defects using cages with the aid of rapid prototyping [J]. The Journal of Arthroplasty, 2013, 28(10): 1770-1775.

[4] ZHANG Y Z, LU S, YANG Y, et al. Design and primary application of computer-assisted, patient-specific navigational templates in metal-on-metal hip resurfacing arthroplasty [J]. The Journal of Arthroplasty, 2011, 26(7): 1083-1087.

[5] LU S, XU Y Q, CHEN G P, et al. Efficacy and accuracy of a novel rapid prototyping drill template for cervical pedicle screw placement [J]. Computer Aided Surgery, 2011, 16(5): 240-248.

[6] DERBY B. Printing and prototyping of tissues and scaffolds [J]. Science, 2012, 338(6109): 921-926.

[7] DING C M, QIAO Z G, JIANG W B, et al. Regeneration of a goat femoral head using a tissue-specific, biphasic scaffold fabricated with CAD/CAM technology [J]. Biomaterials, 2013, 34(28): 6706-6716.

[8] ALBERTI C. Three-dimensional CT and structure models [J]. The British Journal of Radiology, 1980, 53(627): 261-262.

[9] MAVILI M E, CANTER H I, SAGLAM-AYDINATAY $\mathrm{B}$, et al. Use of three-dimensional medical modeling methods for precise planning of orthognathic surgery [J]. The Journal of Craniofacial Surgery, 2007, 18(4): 740-747.

[10] ROSSET A, SPADOLA L, RATIB O. OsiriX: An open-source software for navigating in multidimensional DICOM images [J]. Journal of Digital Imaging, 2004, 17(3): 205-216.

[11] BARBORIAK D P, PADUA A O, YORK G E, et al. Creation of DICOM - Aware applications using ImageJ [J]. Journal of Digital Imaging, 2005, 18(2): 9199. 